\title{
Aspectos psico-espirituales medidos con la FACIT-Sp en pacientes con cáncer estadio IV.
}

\author{
Psycho-spiritual elements evaluated by FACIT-Sp in stage IV cancer patients.

\begin{abstract}
Bruno Pedraz-Petrozzi ${ }^{1, a}$, Juan Diego Mendoza-Saldaña ${ }^{2}$, Astrid Altamirano-Quiroz ${ }^{2, a}$,

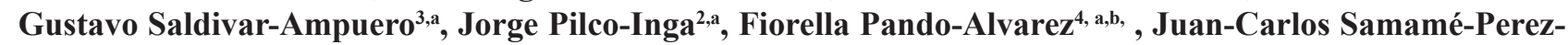
$\operatorname{Vargas}^{4, a, b}$.
\end{abstract}

\section{RESUMEN}

Objetivo: Describir los diferentes elementos psico-espirituales en pacientes con cáncer aplicando la escala Functional Assessment of Chronic Illness Therapy - Spiritual Well Being, FACIT-Sp (Evaluación Funcional de Terapia de Enfermedades Crónicas y Bienestar Espiritual). Métodos: Aplicación de la escala a una muestra de pacientes $(n=$ 100) con diagnóstico de cáncer, estadio IV, del Servicio de Oncología del Hospital Nacional Arzobispo Loayza. El instrumento de 33 preguntas examina cinco áreas: bienestar social (SWB), bienestar emocional (EWB), bienestar funcional (FWB), sentido y paz (MP) y fe (F). Resultados: En la muestra estudiada, el aspecto más resaltante fue el relacionado con $\mathrm{F}$ (puntaje promedio $=13,53 \pm 3,05$, correspondiente al $84,56 \%$ del puntaje máximo posible) y el más bajo fue el vinculado a FWB (puntaje promedio=15,79 $\pm 6,89$, correspondiente al 56,39\% del puntaje máximo posible). Conclusiones: De acuerdo a FACIT-Sp, el aspecto psico-espiritual más desarrollado en los pacientes de la muestra estudiada fue el relativo a la Fe y el menos desarrollado, el vinculado al área de Bienestar Funcional.

PALABRAS CLAVE: Psicooncología, medicina paliativa, espiritualidad, metástasis, neoplasias.

\section{SUMMARY}

Objective: To describe different psycho-spiritual elements in a sample of stage IV cancer patients from a public hospital in Lima, Peru, through the application of the Functional Assessment of Chronic Illness Therapy - Spiritual Well-Being (FACIT-Sp) scale. Methods: Application of FACIT-Sp to a sample of patients $(\mathrm{n}=100)$ diagnosed with stage IV cancer at the Oncology Service of the Hospital Nacional Arzobispo Loayza. The 33-question instrument, designed to evaluate psycho-spiritual elements, analyzes five areas: social well-being (SWB), emotional well-being (EWB), functional well-being (FWB), meaning and peace (MP) and faith (F). Results: The most prominent aspect was $\mathrm{F}$ (average score $=13.53 \pm 3.05$, corresponding to $84.56 \%$ of the maximum possible score), and the lowest was FWB (average score $=15.79 \pm 6.89$, corresponding to $56.39 \%$ of the maximum possible score). Conclusions: According to FACIT-Sp, the most developed psycho-spiritual aspect among the participating patients was the one related to Faith, whereas the least developed, the one linked to the area of Functional Well-Being.

KEYWORDS: Neoplasms, palliative care, metastasis, spirituality, psycho-oncology.

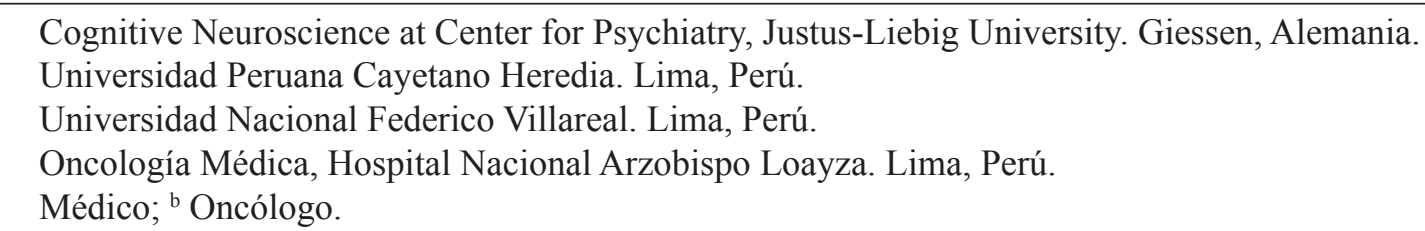




\section{INTRODUCCIÓN}

La Organización Mundial de la Salud (OMS) define "salud" como el estado completo de bienestar físico, mental y social, y no sólo la ausencia de enfermedad (1); bajo este concepto, se deriva el enfoque holístico, que incluye un manejo multidisciplinario y que abarca diversos aspectos de la persona.

En los últimos años, mediante este modelo, es que se han ido modificando los cuidados de los pacientes con diversas enfermedades crónicas, siendo un ejemplo de ello el cáncer (2-4). Esta última enfermedad ha aumentado considerablemente en prevalencia y amenaza con convertirse en la primera causa de morbimortalidad en todo el mundo (5-7). Se estima entonces un aumento pronunciado en la cantidad de personas con la enfermedad, pero sobretodo hay cierta especial atención en aquellos en estadios finales de la enfermedad $(8,9)$. De forma que se pueda mejorar la atención y la calidad de vida (QoL), se han ido orientando diversas acciones hacia un esquema con enfoque holístico en pacientes con cáncer $(10,11)$. Según este enfoque, estos pacientes requieren de refuerzos en diversos aspectos, como en aquellos centrados en la persona, incluidos elementos psico-espirituales $(2,12)$. A pesar de la relevancia del tema en el enfoque del cuidado del paciente, no existen muchas publicaciones que se centren en medir o analizar los efectos psico-espirituales y el impacto en la calidad de vida de este grupo de pacientes $(13,14)$. De la literatura disponible, existen algunas escalas validadas que examinan elementos psico-espirituales y que los definen bajo los siguientes constructos: propósito y sentido de vida, aceptación de la muerte, satisfacción con la vida, paz con "dios" o con un ser superior, preparación para la muerte, religión y opinión/reflexión acerca de la vida $(15,16)$.

Dentro de las escalas empleadas específicamente para evaluar elementos psico-espirituales en pacientes con enfermedades crónicas cómo el cáncer, se encuentra la denominada "Evaluación Funcional de Terapia de Enfermedades Crónicas y Bienestar Espiritual" (FACIT - Sp por las siglas del inglés "Functional Assessment of Chronic Illness Therapy - Spiritual Well Being”). Este instrumento define y evalúa dichos elementos psicoespirituales desde tres principales dimensiones: sentido, paz y fe. Esta escala ha sido ampliamente utilizada en personas con cáncer, inicialmente para una compresión holística de la QoL. Además, el instrumento FACIT-Sp ha sido evaluado y validado en países como Estados Unidos, Japón, Puerto Rico y Colombia, entre otros (17-20).
En nuestro país la incidencia de cáncer es elevada en comparación con otros países de la región (21). Adicionalmente, existen dos estudios que sugieren la necesidad de enfocar el entendimiento de la enfermedad hacia un modelo integral, donde se incluyan elementos psico-espirituales en para un manejo multidisciplinario de dichos pacientes $(22,23)$. Por estos motivos, hemos considerado necesario realizar un estudio en pacientes con cáncer en estadio IV, que tenga como principal objetivo describir el panorama de elementos psico-espirituales según la escala FACIT-Sp, de forma preliminar a un estudio de validación, en una muestra de pacientes de un hospital universitario de Lima-Perú.

\section{MATERIAL Y MÉTODOS}

El presente es un estudio descriptivo. Para la recolección de datos concerniente a los elementos psico-espirituales, se empleó la escala FACIT-Sp. Esta escala ya ha sido validada en diversos países de habla hispana y consta actualmente con una versión oficial en castellano latinoamericano (24).

La escala FACIT-Sp cuenta originalmente con seis áreas: estado físico general de salud (PWB), bienestar social (SWB), bienestar emocional (EWB), bienestar funcional (FWB), sentido y paz (MP), y fe (F). Para poder evaluar exclusivamente elementos psicoespirituales, se removió PWB y permanecieron las siguientes áreas: SWB, EWB, FWB, MP y F. Las áreas incluidas evalúan elementos del ambiente familiar y social, del estado emocional, de la capacidad de funcionamiento personal y de la espiritualidad (p.ej. sentido/trascendencia, paz y fe). Adicionalmente, se convocó a un grupo de expertos del Hospital Nacional Arzobispo Loayza (HNAL) -los cuales no tuvieron ninguna relación con los investigadores del área de oncología de dicho centro- para realizar reuniones periodicas ( 5 días/semana, 2 semanas) con la finalidad de valorar la factibilidad y comprensión de la escala por parte de los pacientes que acuden a los establecimientos de salud del estudio.

Después de corroborada la factibilidad y la comprensión, se elaboró un formato de recolección de datos que incluyó lo siguiente: (a) variables sociodemográficas (p.ej. sexo, edad, ocupación, etc.), (b) variables relacionadas con el diagnóstico oncológico (p.ej. diagnostico, lugares de metástasis, tratamiento, etc.) y (c) características psico-espirituales, es decir las áreas incluidas de la escala FACIT-Sp (SWB, EWB, FWB, MP y F). 
En relación a los detalles técnicos de la escala empleada, cada pregunta fue construida para disponer de respuestas de alternativa multiple, siguiendo el modelo de Likert ( 0 para el puntaje más bajo y 4 para el más alto). Con respecto a los puntajes, se siguió el manual de aplicación original del FACIT-Sp. En este se define cómo 0 al puntaje más bajo por cada área. Los puntajes más altos variaron entre áreas (28 para SWB y FWB, 24 para EWB, 32 para MP y 16 para F). Con respecto a los puntajes de las diferentes áreas, según el protocolo de aplicación (25) rige lo siguiente: a mayor puntaje, mejor componente psico-espiritual. Debido que cada componente tiene puntajes máximos diferentes, se expresaron los resultados también en porcentajes, dividiendo el puntaje promedio obtenido de la muestra entre el puntaje máximo posible del área correspondiente de forma que se pueda comparar los resultados entre los diferentes componentes psicoespirituales.

Posteriormente, se aplicó la encuesta en pacientes. Los criterios de inclusión fueron:

a. Ser pacientes del HNAL, ya sea hospitalizados (en los servicios de medicina interna, cirugía y ginecología; y con interconsulta respectiva a oncología) o ambulatorios (pertenecientes al servicio de consultorio de Oncología Médica).

b. Sujetos mayores de 18 años y con el diagnóstico de estadio IV para cáncer. El diagnóstico de neoplasia maligna metastásica debía ser confirmado con histología de metástasis, independientemente del sitio primario o secundario de la enfermedad.

c. El previo llenado del consentimiento informado.

Aquellos pacientes que no deseaban libremente participar en el estudio, pacientes con síntomas psicóticos, delusionales o hemodinámicamente descompensados, además de pacientes menores de 18 años, fueron excluidos del estudio.

Este estudio fue aprobado por el Comite de Ética del HNAL. Los consentimientos informados fueron redactados y aplicados bajo los principios de la Declaración de Helsinki. Se garantizó en todo momento el anonimato de los participantes mediante codificación. Los resultados fueron analizados con SPSS versión 24.0.

\section{RESULTADOS}

Se reclutaron en total 100 pacientes, 88 de los cuales eran ambulatorios y 12 se hallaban hospitalizados, todos tenían diagnóstico de cáncer estadio IV. Los resultados de la aplicación del cuestionario FACIT-Sp de acuerdo a las áreas a estudiar fueron los siguientes:

Variables sociodemográficas: Los datos correspondientes a las variables socio-económicas se encuentran detalladas en la tabla 1. La muestra evaluada constó de 1,5 veces más mujeres que hombres y la edad promedio de todos los participantes fue de 59,36 años. La mayoría de los participantes finalizaron la escuela secundaria, se definieron a sí mismos como "cristiano católicos" y provenían del distrito de San Juan de Lurigancho. Respecto a la información laboral de los participantes, se obtuvo que la mayoría no trabajaban. De aquellos participantes que presentan actividades laborales, más de la mitad trabajaba en los quehaceres del hogar. De los participantes que se declararon a sí mismos como "jefe de familia", se halló que un 70\% de ellos fueron mujeres.

Variables relacionadas con el diagnóstico oncológico: El estado funcional de la mayoría de los pacientes estadio IV enrolados fue "adecuado", según los criterios de la escala ECOG ("Eastern Cooperative Oncology Group") de la OMS para disfuncionalidad de enfermedad $(26,27)$. La información sobre el estado funcional ECOG se encuentra señalada en la tabla 2. La gran mayoría de los pacientes fue diagnosticada de cáncer de mama, seguido de cáncer gástrico. De los participantes, un $84 \%$ de los sujetos tuvieron metástasis en menos de dos lugares o en dos órganos diferentes (paciente oligometastásico). Por otro lado, un 11\% de los participantes reportaron metástasis en más de dos órganos (paciente plurimetastásico). Los órganos que más afectación metastásica presentaron, fueron: hueso, hígado y pulmones. Con relación al tratamiento recibido, la mayoría de los participantes han recibido quimioterapia anteriormente. Por otro lado, la mayoría de los participantes se encontraban en tratamiento quimioterapéutico al momento del reclutamiento. Más información sobre los diagnósticos, lugares de metastasis y tratamiento se encuentran en la tabla 3.

Características psico-espirituales: Utilizando la versión revisada en castellano del FACIT-Sp, se evaluaron a los participantes que libremente accedieron a participar en el estudio. Las preguntas exploraron los siguientes ítems: SWB (7 preguntas), EWB (5 preguntas), FWB (7 preguntas), MP (8 preguntas) y F (4 preguntas). Los resultados promedio de los participantes fueron divididos entre el puntaje más alto posible según el área y representados con porcentajes (tabla4). 
Tabla 1. Características socio-demográficas de la muestra $(n=100)$.

\begin{tabular}{lcr}
\hline Edad (en años) & & $59,36 \pm 1$ \\
\hline Sexo & Frecuencia & Porcent \\
$\quad$ Participantes femeninos & 72 & $72 \%$ \\
$\quad$ Participantes masculinos & 28 & $28 \%$ \\
Religión & & \\
$\quad$ Cristiana católica & 63 & $63 \%$ \\
Cristiana no católica & 29 & $29 \%$ \\
Ateo & 1 & $1 \%$ \\
Agnóstico & 1 & $1 \%$ \\
$\quad$ No precisa & 6 & $6 \%$ \\
Distrito/lugar de procedencia & &
\end{tabular}

Distrito/lugar de procedencia

San Juan de Lurigancho

Lima

9

Cercado de Lima

8

San Martín de Porres

Breña

5

Los Olivos

5

Ate

Chorrillos

Otros

$5 \%$

$5 \%$

$4 \%$

$4 \%$

Grado de educación

Secundaria completa

Primaria incompleta

Secundaria incompleta

Solo refiere "secundaria"

Solo refiere "primaria"

Solo refiere "superior"

Superior técnica completa

$5 \quad 5 \%$

Analfabeto

4

Primaria completa

$3 \quad 3 \%$

Superior técnica incompleta

1

Superior universitaria incompleta

1

1

Superior universitaria completa

$1 \%$

No brinda información

“Usted trabaja?”

Sí 
Aspectos psico-espirituales medidos con la FACIT-Sp en pacientes con cáncer estadio IV.

Tabla 1 (cont). Características socio-demográficas de la muestra $(\mathrm{n}=100)$.

\begin{tabular}{|c|c|c|}
\hline & Frecuencia & Porcentaje \\
\hline No precisa & 2 & $2 \%$ \\
\hline \multicolumn{3}{|l|}{ “¿A qué se dedica?" } \\
\hline Su casa & 59 & $59 \%$ \\
\hline Desempleado & 20 & $20 \%$ \\
\hline Comerciante & 3 & $3 \%$ \\
\hline Taxista & 2 & $2 \%$ \\
\hline Chofer (no taxista) & 2 & $2 \%$ \\
\hline Contador & 1 & $1 \%$ \\
\hline Niñera/Niñero & 1 & $1 \%$ \\
\hline Ambulante & 1 & $1 \%$ \\
\hline Acabados (interiores) & 1 & $1 \%$ \\
\hline Costurero/Costurera & 1 & $1 \%$ \\
\hline Publicidad & 1 & $1 \%$ \\
\hline No precisa & 8 & $8 \%$ \\
\hline \multicolumn{3}{|l|}{ "¿Vive solo?" } \\
\hline Sí & 12 & $12 \%$ \\
\hline No & 85 & $85 \%$ \\
\hline No precisa & 3 & $3 \%$ \\
\hline \multicolumn{3}{|l|}{ “¿Es usted jefe de familia?” } \\
\hline Sí & 30 & $30 \%$ \\
\hline No & 65 & $65 \%$ \\
\hline No precisa & 5 & $5 \%$ \\
\hline \multicolumn{3}{|c|}{ “:Tiene o ha tenido familiares con cáncer?” } \\
\hline Sí & 45 & $45 \%$ \\
\hline No & 52 & $52 \%$ \\
\hline No precisa & 3 & $3 \%$ \\
\hline
\end{tabular}

Tabla 2. Estado funcional de los participantes enrolados según la escala ECOG.

\begin{tabular}{lcc}
\hline & Frecuencia & Porcentaje \\
\hline ECOG 0 & 11 & $11,30 \%$ \\
ECOG 1 & 43 & $44,30 \%$ \\
ECOG 2 & 27 & $27,80 \%$ \\
ECOG 3 & 13 & $13,40 \%$ \\
ECOG 4 & 3 & $3,10 \%$ \\
\hline
\end{tabular}


Tabla 3. Diagnóstico, lugares de metástasis y tratamientos empleados.

\begin{tabular}{|c|c|c|}
\hline & Frecuencia & Porcentaje \\
\hline \multicolumn{3}{|l|}{ Diagnóstico Oncológico } \\
\hline Cáncer de Mama & 23 & $23 \%$ \\
\hline Cáncer Gástrico & 15 & $15 \%$ \\
\hline Cáncer de Cérvix & 13 & $13 \%$ \\
\hline Cáncer de Pulmón & 5 & $5 \%$ \\
\hline Cáncer de Colon & 5 & $5 \%$ \\
\hline Cáncer de Ovario & 5 & $5 \%$ \\
\hline Cáncer de Próstata & 5 & $5 \%$ \\
\hline Cáncer de Recto & 4 & $4 \%$ \\
\hline Cáncer de Canal Anal & 3 & $3 \%$ \\
\hline Tumor del Sistema Nervioso Central & 2 & $2 \%$ \\
\hline Cáncer de Endometrio & 2 & $2 \%$ \\
\hline Cáncer de Páncreas & 2 & $2 \%$ \\
\hline No especificado & 2 & $2 \%$ \\
\hline Otros & 14 & $14 \%$ \\
\hline Localización de la metástasis & Frecuencia & Porcentaje \\
\hline Huesos & 19 & $19 \%$ \\
\hline Hígado & 18 & $18 \%$ \\
\hline Pulmón & 13 & $13 \%$ \\
\hline Pleura & 10 & $10 \%$ \\
\hline Sistema Nervioso Central & 7 & $7 \%$ \\
\hline Ganglios Retroperitoneales & 6 & $6 \%$ \\
\hline Peritoneo & 4 & $4 \%$ \\
\hline Ganglios (sin especificar zona) & 3 & $3 \%$ \\
\hline Músculo estriado esquelético & 2 & $2 \%$ \\
\hline Piel & 2 & $2 \%$ \\
\hline Ganglios Mesentéricos & 2 & $2 \%$ \\
\hline Otros & 14 & $14 \%$ \\
\hline Tratamiento previo & Frecuencia & Porcentaje \\
\hline Solo quimioterapia & 57 & $57 \%$ \\
\hline Quimioterapia con Cirugía & 9 & $9 \%$ \\
\hline No especificado & 8 & $8 \%$ \\
\hline Quimioterapia, Radioterapia y Cirugía & 7 & $7 \%$ \\
\hline Ninguno & 4 & $4 \%$ \\
\hline Quimioterapia con Hipertermia & 4 & $4 \%$ \\
\hline Quimioterapia con Radioterapia & 3 & $3 \%$ \\
\hline Cirugía & 2 & $2 \%$ \\
\hline
\end{tabular}


Aspectos psico-espirituales medidos con la FACIT-Sp en pacientes con cáncer estadio IV.

Tabla 3 (cont). Diagnóstico, lugares de metástasis y tratamientos

\begin{tabular}{lcc}
\hline & Frecuencia & Porcentaje \\
\hline Tratamiento Paliativo & 2 & $2 \%$ \\
Quimioterapia, Radioterapia y Braquiterapia & 2 & $2 \%$ \\
TARGA & 1 & $1 \%$ \\
Quimioterapia, PCL, Radioterapia y Cirugía & 1 & $1 \%$ \\
Tratamiento Actual & Frecuencia & Porcentaje \\
Quimioterapia sola & 46 & $46 \%$ \\
Tratamientos paliativos para el dolor & 20 & $20 \%$ \\
Tratamiento paliativo no especificado & 7 & $7 \%$ \\
Observación & 6 & $6 \%$ \\
Hipertermia & 5 & $5 \%$ \\
Ninguno & 4 & $4 \%$ \\
Quimioterapia con sintomáticos & 3 & $3 \%$ \\
Radioterapia & 2 & $2 \%$ \\
Cirugía & 2 & $2 \%$ \\
Paliativo: Antibioticoterapia con radioterapia & 1 & $1 \%$ \\
Paliativo: Drenaje torácico bilateral con radioterapia & 1 & $1 \%$ \\
Paliativo: interferón & 1 & $1 \%$ \\
TARGA & 1 & $1 \%$ \\
Paliativo: Drenaje torácico bilateral & 1 & $1 \%$ \\
\hline
\end{tabular}

Tabla 4. Puntajes y porcentajes del FACIT-Sp en la muestra.

\begin{tabular}{lccc}
\hline Áreas & Puntajes $(\mathbf{m} \pm \mathbf{D E})$ & Puntaje máximo & Porcentajes* \\
\hline SWB & $19,07 \pm 5,82$ & 28 & $68,11 \%$ \\
EWB & $16,44 \pm 5,39$ & 24 & $68,50 \%$ \\
FWB & $15,79 \pm 6,89$ & 28 & $56,39 \%$ \\
MP & $21,57 \pm 4,73$ & 32 & $67,41 \%$ \\
F & $13,53 \pm 3,05$ & 16 & $84,56 \%$ \\
\hline *Dado que las diferentes áreas evaluadas miden elementos distintos, se calcularon porcentajes en \\
relación al puntaje máximo por cada área evaluada (es decir: Porcentajes = Media (Puntaje)/Puntaje \\
máximo posible) para poder comparar y determinar cuál de las áreas es la más/menos reforzada
\end{tabular}

\section{DISCUSIÓN}

Los resultados obtenidos en el presente estudio pueden analizarse de acuerdo a las tres áreas estudiadas: elementos socio-demográficos, elementos clínico-oncológicos y elementos vinculados al factor psico-espiritual.

Elementos socio-demográficos: La información obtenida en este estudio es compatible con la información reportada previamente por el HNAL, según los análisis epidemiológicos del 2014 y del 2011, en el que se aprecia que la mayoría de pacientes pertenecían al sexo femenino, no habían completado estudios escolares, provenían de la zona norte de Lima Metropolitana, profesaban la religión católica, estaban desempleados y vivían con una persona adicional para poder ayudarles en sus quehaceres diarios debido a tener algún grado de disfunción secundario al cáncer $(28,29)$. Llama la atención que solo la tercera parte se declaró como jefe de familia, y de este porcentaje, la mayoría eran mujeres. 
Elementos clínico-oncológicos: Al analizar las variables clínico-oncológicas, se vio que los diagnósticos oncológicos más comunes fueron: neoplasia de estómago, mama y cérvix $(28,29)$. Además, casi la mitad de los pacientes evaluados presentaba un grado de función adecuado según la escala ECOG. En ese sentido, nuestro estudio presenta similaridades con otros trabajos en la misma línea de la evidencia. Un ejemplo de ello es un estudio realizado por Noguchi et al., (19), en el cual la mayoría de pacientes presentaban una escala funcional según ECOG similar a la obtenida en nuestro estudio. Sin embargo, y en comparación con el estudio de Noguchi et al., (19), nuestro estudio presenta ciertas diferencias, como por ejemplo el tamaño muestral, el diagnóstico oncológico más frecuente (cáncer de próstata), así como también el tipo de tratamiento que recibían al momento del estudio (radioterapia). En otro estudio, realizado por Peterman et al., (17), en el que se evaluó la escala FACIT-Sp también en una población oncológica, la mayoría de los pacientes presentaron un ECOG similar a lo obtenido en nuestro estudio. No obstante, los pacientes evaluados se hallaban en estadio 1. En relación a los patrones de diseminación metstásica, hemos encontrado diferencias con la literatura. Nuestros hallazgos reportan que el hueso es el lugar más común de afectación metastásica, seguido del hígado. Por el contrario, en la mayoría de los estudios se encuentra que la zona hepática fue la más común de asiento de secundarismo (30).

\section{Elementos vinculados al factor psico-espiritual:} En relación nuestros resultados del FACIT-Sp relacionados al área psico-espiritual, se observó que los ítems vinculados a la $\mathrm{F}$ fueron los más fortalecidos y que los ítems vinculados al FWB los menos favorecidos. Nuestros resultados coinciden con un estudio realizado en población colombiana de pacientes con cáncer, donde la escala FACITSp fue aplicada (24). En ambos resultados existe predominancia de la área $\mathrm{F}$ sobre otros elementos de la escala FACIT-Sp (24). Estas similitudes pueden sugerir un posible factor religioso preponderante en nuestra región y que, por multiples motivos, se encuentra incluida en la cultura. Además, nuestros resultados coinciden curiosamente con un estudio realizado en población árabe predominantemente musulmana donde se reportan altos valores en las áreas de F y MP en pacientes oncológicos (31). En este caso, vemos que la importancia para los pacientes oncológicos de espiritualidad se ve reflejada en los altos valores en las áreas relacionadas con la parte espiritual o religiosa. Finalmente, nuestros resultados, a la par con las características socio-demográficas de nuestro estudio, podrían sugerir que existe un factor religioso-espiritual fuerte, a pesar de las carencias de nuestra población, y que ello podría servir como base para un contrapeso emocional en el contexto de la salud-enfermedad.

Limitaciones: En nuestro estudio hemos hallado dos limitantes principalmente. La primera fue el tamaño muestral. Esto se debió principalmente al corto periodo de captación de pacientes. Además, porque nuestra población se obtuvo de un hospital general y no de un establecimiento dedicado solo al manejo oncológico, como lo sería un instituto (32). Segundo, la falta de estudios de validación de constructos psicoespirituales en nuestro país, a pesar de ya existir estudios que han estudiado una versión castellana en otros lugares de nuestra región latinoamericana (p.ej. Colombia) (24). En ese sentido, se presentaron dificultades en la comprensión de algunos terminos de ítems de la escala, sobretodo "armonía interna", "sentido de vida", "propósito" (relacionados con los ítems del componente psico-espiritual). Ello produjo cierta demora para contestar el cuestionario y en algunos casos respuestas heterogéneas, dependiendo cómo entendía cada paciente la pregunta. Al no haber uniformidad con las reacciones y al ver que los pacientes preguntaban sobre el significado de las palabras, se puede inferir que el lenguaje representó una limitante. No obstante, los evaluadores estuvieron en todo momento en la recolección de datos y estuvieron capacitados para responder las preguntas de los pacientes, de forma que se comprendiesen los ítems. Por último, se podría decir que otro intercurrente fue el hecho de la modificacion del cuestionario FACIT-Sp, obviando los ítems para la evaluación física, obteniendo una escala mayormente centrada en características psico-espirituales ( $f e$, sentido de trascendencia y paz). Nuestro interés de modificar el FACIT-Sp se dio con el hecho de que nuestra pregunta de investigación se orientaba principalmente en encontrar dichas características (33).

Perspectivas Futuras: Muchos de los estudios psicométricos en la actualidad están buscando diferentes definiciones para diversos constructos. Este sería el caso de los elementos psico-espirituales. Cuanto más haya interés en definir elementos psicoespirtuales, específicamente en pacientes con estadio IV de cáncer, más cuestionarios se escribirán y el concepto a buscar será más sólido para así poder intervenir de forma holística en la población, tal cual busca la OMS en su concepto de salud. De este modo 
el abordaje orientado al cáncer cambiaría incluso desde su visión de salud pública, el cual hoy en día se enfoca en la prevención y tratamiento médico, pero no en el apoyo psico-espiritual, lo cual al parecer es una necesidad inminente.

El principal objetivo de nuestro estudio fue describir elementos psico-espirituales de pacientes de un hospital general de Lima-Perú, afectados por una enfermedad crónica/terminal (cáncer estadio IV) empleando la escala FACIT-Sp. Consideramos importante el reportar estos resultados como un estudio descriptivo para resaltar la importancia de elementos psico-espirituales en pacientes con enfermedades avanzadas como el caso del cáncer, pues es un ítem que habitualmente no se toma en cuenta para el análisis ni para el diseño de estudios clínicos en esta población y otras semejantes. De forma paralela, cabe mencionar que de acuerdo a nuestra revisión bibliográfica, aún no se ha reportado información sobre psico-espiritualidad empleando el FACIT-Sp en pacientes peruanos de un hospital general ni con diagnóstico de cáncer u otro. Por lo que se puede decir que el área más reforzada en este estudio fue la relacionada con la $\mathrm{F}$, siendo la primera característica descrita en la población peruana de hospital general y por ende merece mayor análisis.

En conclusión, nuestros resultados evidencian que la $f e$ (ítem definido en la escala FACIT-Sp) es el aspecto que más destaca en el concepto de psico-espiritualidad en pacientes peruanos con cáncer metastásico. En contraste, bienestar funcional fue el área con menos porcentaje en nuestra muestra, y por ende el que menos destacó entre los otros ítems. Finalmente, creemos que futuros estudios deben de orientarse a poder estudiar estas características en otros grupos de pacientes con cáncer y quizás en otras regiones de nuestro país ya que como sabemos existen diversos factores (p.ej. culturales, sociales, económicos, etc.) que pueden influir en la espiritualidad y en la percepción del fenómeno salud-enfermedad.

Conflicto de Intereses: Los autores de este manuscrito declaran no tener ningún conflicto de interés.

Agradecimientos: Los autores de este articulo agradecen al personal médico y sanitario del Hospital Nacional Arzobispo Loayza, así como al personal del Servicio de Oncología de la misma institución. Agradecemos a su vez al Dr. Fernando Runzer por el apoyo brindado y los comentarios relacionados a la metodología del artículo.

\section{Correspondencia:}

Dr. Bruno Pedraz Petrozzi.

Klinikstrasse 36, D-35392 Giessen, Hessen, Alemania. Telefono: 496419945773

Correo electrónico: Bruno.Pedraz@med.uni-giessen. de

\section{REFERENCIAS BIBLIOGRÁFICAS}

1. World Health Organization. Official records of the World Health Organization: Summary report on proceedings minutes and final acts of the international health conference. Int Heal Conf. 1946; 2:1-143.

2. Cadet T, Davis C, Elks J, Wilson P. A holistic model of care to support those living with and beyond cancer. Healthcare. 2016; 4(4):88-96.

3. Phelps AC, Lauderdale KE, Alcorn S, Dillinger J, Balboni MT, Van Wert M, et al. Addressing spirituality within the care of patients at the end of life: Perspectives of patients with advanced cancer, oncologists, and oncology nurses. J Clin Oncol. 2012; 30(20): $2538-2544$.

4. Taylor EJ, Mamier I. Spiritual care nursing: What cancer patients and family caregivers want. J Adv Nurs. 2005; 49(3): 260-267.

5. Siegel RL, Miller KD, Jemal A. Cancer statistics 2019. CA Cancer J Clin. 2019; 69(1): 7-34.

6. Torre LA, Bray F, Siegel RL, Ferlay J, Lortet-Tieulent J, Jemal A. Global cancer statistics, 2012. CA Cancer J Clin. 2015; 65(2): 87-108.

7. Parkin DM. Global cancer statistics in the year 2000. Lancet Oncol. 2001; 2(9): 533-543.

8. Small AC, Tsao C-K, Moshier EL, Gartrell BA, Wisnivesky JP, Godbold JH, et al. Prevalence and characteristics of patients with metastatic cancer who receive no anticancer therapy. Cancer. 2012;118(23):5947-5954.

9. Miller KD, Siegel RL, Lin CC, Mariotto AB, Kramer $\mathrm{JL}$, Rowland $\mathrm{JH}$, et al. Cancer treatment and survivorship statistics, 2016. CA Cancer J Clin. 2016;66(4):271-289.

10. World Health Organization. Cancer: WHO definition of palliative care. Geneva: World Health Organization;1990. (Citado el 3 de marzo del 2020) Disponible en: https://www.who.int/cancer/palliative/ definition/en/

11. Davies E, Higginson I. Better palliative care for older people: Report from the Regional Office for Europe. Geneva: World Health Organization; 2004. pp. 1-43.

12. Connor SR, Sepulveda-Bermedo MC. The global atlas of palliative care at the end of life: An advocacy tool. Report from the World Health Organization. Geneva: World Health Organization; 2014. Pp. 1-111.

13. Kandasamy A, Chaturvedi S, Desai G. Spirituality, 
distress, depression, anxiety, and quality of life in patients with advanced cancer. Indian J Cancer. 2011;48(1):55-59.

14. Clay KS, Talley C, Young KB. Exploring spiritual well being among survivors of colorectal and lung cancer. J Relig Spiritual Soc Work Soc Thought. 2010;29(1):14-32.

15. Albers G, Echteld MA, De Vet HCW, OnwuteakaPhilipsen BD, Van Der Linden MHM, Deliens L. Content and spiritual items of quality-of-life instruments appropriate for use in palliative care: A review. J Pain Symptom Manage. 2010; 40(2):290300.

16. Brady MJ, Peterman AH, Fitchett G, Mo M, Cella D. A case for including spirituality in quality of life measurement in oncology. Psychooncology. 1999;8(5):417-428.

17. Peterman AH, Fitchett G, Brady MJ, Hernandez L, Cella D. Measuring spiritual well-being in people with cancer: The functional assessment of chronic illness therapy - spiritual well-being scale (FACITSP). Ann Behav Med. 2002;24(1):49-58.

18. Sánchez R, Sierra F, Zárate K. ¿Son la religiosidad y la espiritualidad dimensiones diferentes? Rev Colomb Cancerol. 2014;18(2):62-68.

19. Morita S, Aihara O, Tsujii H, Shimozuma K, Matsushima E, Noguchi W, et al. Reliability and validity of the Functional Assessment of Chronic Illness Therapy-Spiritual (FACIT-Sp) for Japanese patients with cancer. Support Care Cancer. 2004;12(4):240-245.

20. Canada AL, Murphy PE, Fitchett G, Peterman AH, Schover LR. A 3-factor model for the FACIT-Sp. Psychooncology. 2008;17(9):908-916.

21. Ramos W, Venegas D, Medina J, Guerrero P, Cruz A. Análisis de la Situación de Cáncer en el Perú.. Lima, Perú: Ministerio de Salud; 2013. Pp. 1-108.

22. Valle R, Zúñiga M, Tuzet C, Martínez C, De La Jara J, Aliaga R, et al. Sintomatología depresiva y calidad de vida en pacientes mujeres con cáncer de mama. Anales de la Fac Medicina. 2006;67(4):327-332.

23. Salas-Pardo J. Calidad de vida del paciente oncológico terminal asistente a una unidad de terapia especializada. Cuid Salud. 2014;1(1):1-10.

24. Sierra FA. Validación para la utilización en Colombia de la escala FACIT-Sp para evaluación de bienestar espiritual en pacientes con cáncer. Tesis de Grado. Bogota: Universidad Nacional de Colombia; 2012. 1-115 pp.
25. Bredle JM, Salsman JM, Debb SM, Arnold BJ, Cella D. Spiritual well-being as a component of healthrelated quality of life: The Functional Assessment Of Chronic Illness Therapy-Spiritual Well-Being Scale (FACIT-Sp). Religions. 2011; 2(1) 77-94

26. Oken MM, Creech RH, Tormey DC, Horton J, Davis TE, McFadden ET CP. Toxicity and response criteria of the Eastern Cooperative Oncology Group. Am J Clin Oncol. 1982; 5(6): 649-655.

27. Oken M, Creech R, Tormey D, Horton J, Davis T, McFadden E, et al. Toxicity and response criteria of the Eastern Cooperative Oncology Group. Am J Clin Oncol.1982; 5(6):649-55.

28. Ministerio de Salud. Informe de la Oficina de Epidemiología y Salud Ambiental: Análisis de Situación de Salud 2014- Hospital Nacional "Arzobispo Loayza". Lima, Perú: Ministerio de Salud; 2014. Pp. 1-287.

29. Ministerio de Salud. Informe de la Oficina de Epidemiología y Salud Ambiental. Análisis de Situación de Salud 2011 - Hospital Nacional "Arzobispo Loayza". Lima, Perú: Ministerio de Salud; 2012. pp. 1-221.

30. Budczies J, von Winterfeld M, Klauschen F, Bockmayr M, Lennerz JK, Denkert C, et al. The landscape of metastatic progression patterns across major human cancers. Oncotarget. 2015;6(1):570583.

31. Lazenby M, Khatib J, Al-Khair F, Neamat M. Psychometric properties of the Functional Assessment of Chronic Illness Therapy-Spiritual Well-being (FACIT-Sp) in an Arabic-speaking, predominantly Muslim population. Psychooncology. 2013;22(1):220-227.

32. Morales PV. Tamaño necesario de la muestra: ¿Cuántos sujetos necesitamos? Madrid: Universidad Pontifica Comillas; 2012. (Citado el 3 de marzo del 2020) Disponible en: http://data.evalua.cdmx.gob. mx/docs/gral/taller2015/S0202EAC.pdf

33. Munoz AR, Salsman JM, Stein KD, Cella D. Reference values of the Functional Assessment of Chronic Illness Therapy-Spiritual Well-Being: A report from the American Cancer Society's studies of cancer survivors. Cancer. 2015; 121(1): 1838-1844.

Recibido: 09/07/2019

Aceptado: 02/03/2020 